\title{
Correspondence
}

Korean J Ophthalmol 2022;36(1):74-75

https://doi.org/10.3341/kjo.2021.0118

\section{Incarceration of Ciliary Process at Sclerotomy Site after Cataract Sur- gery in Trabeculectomized Eye: A Case Report}

\section{Dear Editor,}

Cataract surgery on a trabeculectomized eye should be performed with caution, since the dynamics of the anterior chamber (AC) can be adversely affected [1]. We describe herein a case of ciliary process incarceration at the sclerotomy site in cataract surgery following trabeculectomy.

A 55-year-old Korean male underwent phacoemulsification of the crystalline lens and intraocular lens (IOL) implantation in the left eye due to aggravated cataract after intraocular surgery. Six months previously, he had undergone an uncomplicated trabeculectomy for uncontrolled intraocular pressure (IOP) on the left eye due to primary open-angle glaucoma. The IOP was controlled to between 6 and $8 \mathrm{mmHg}$ after the trabeculectomy without any noticeable complications. Six months postoperatively, the patient complained of decreased visual acuity (VA) in the operated eye. Aggravation of the cataract in his left eye was notable, dropping his VA from 120 / 200 to 80 / 200 .

Cataract extraction was performed under sub-Tenon's block using phacoemulsification of the crystalline lens. During the phacoemulsification, the $\mathrm{AC}$ became temporarily shallowed by a sudden rise of posterior chamber pressure, but the cataract was extracted without any complication, the IOL being placed in the capsular bag. On day 8 postoperatively, a slit-lamp examination revealed the well

Received: July 22, 2021 Final revision: October 2, 2021

Accepted: November 8, 2021 placed IOL in the capsular bag with deep AC. The IOP had risen to $20 \mathrm{mmHg}$ with a decrease in size of the filtering bleb. On gonioscopy, ciliary body (CB) incarceration to the internal ostium at the sclerotomy site was observed (Fig. 1). Argon laser photocoagulation (spot size, $500 \mu \mathrm{m}$; duration, 0.5 seconds; power, $200 \mathrm{~mW}$ ) was applied to dislodge the incarceration but failed. The patient was recommended for surgical correction but refused it. Instead, a topical medication (Cosopt; Dorzolamide-Timolol fixed combination; Santen, Osaka, Japan) was applied, and the IOP was maintained within the range of 10 to $14 \mathrm{mmHg}$ for 6 months thereafter.

Trabeculectomy followed by cataract surgery significantly increases the risk of bleb failure. One explanation is that $\mathrm{AC}$ inflammation caused by cataract surgery upregulates fibrogenic cytokines in the aqueous humor, leading to excessive scarring at the conjunctival or scleral flap interface [2]. However, to the best of our knowledge, there have been no reports of $\mathrm{CB}$ incarceration at the trabeculectomy

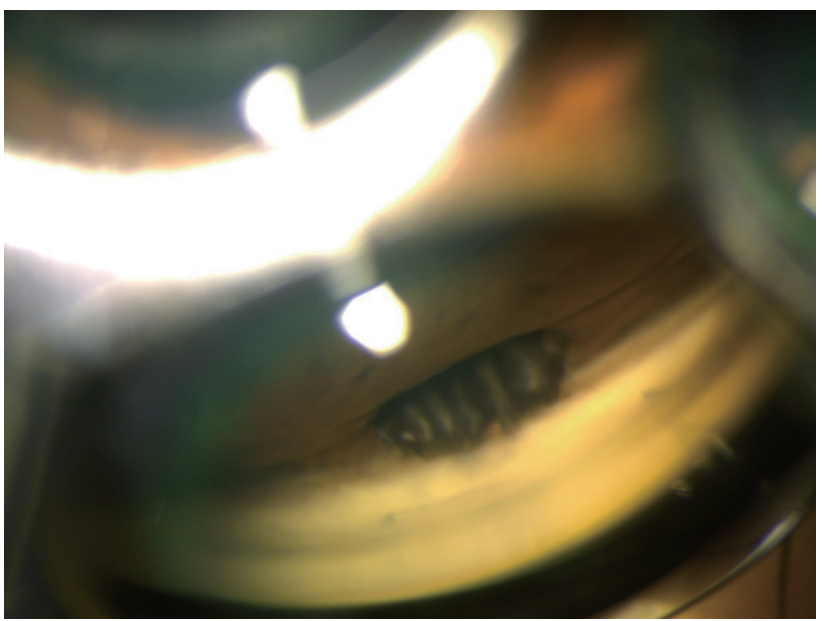

Fig. 1. On day 8 after cataract surgery, ciliary body incarceration to internal ostium of sclerotomy site was observed on gonioscopic examination. Informed consent for publication of the research details and clinical images was obtained from the patient. 
site in patients who had undergone cataract surgery following trabeculectomy. There are, however, a number of reports of early-postoperative $\mathrm{CB}$ incarceration after trabeculectomy without cataract surgery [3-5]. Saari and Heikkila [3] reported six cases of $\mathrm{CB}$ incarceration in the trabeculectomy fistula. Among those cases, none had arisen in the aftermath of cataract surgery following trabeculectomy. Moreover, unlike our case wherein sclerotomy was performed at the trabecular meshwork level, all six of those cases had been performed by Watson's fistulizing technique so that the sclerotomy site could be at the level of the $\mathrm{CB}$ posterior to the scleral spur.

Since there was no excessive intraocular inflammation postoperatively, the elevation of posterior chamber pressure during phacoemulsification is thought to have been the cause of the anterior rotation and CB incarceration. A large iridectomy with or without evident pre-existing zonular damage, especially as it was near the area of the iridectomy site of the trabeculectomy, might have acted as a route for fluid misdirection during the phacoemulsification, thereby increasing the posterior chamber pressure and shallowing the AC. This altered flow and rise of posterior pressure could have incurred anterior rotation of the ciliary process and iris root, eventually leading to the $\mathrm{CB}$ incarceration at the sclerotomy site. However, although the CB incarceration had arisen intraoperatively, surgical repair would be better done as a fallback operation, because the posterior pressure was still expected to be high at the end of the surgery, and there would be a risk of bleeding consequent upon direct manipulation of the $\mathrm{CB}$.

High-volume fluid input during cataract surgery can temporarily increase posterior chamber pressure. Especially in trabeculectomized eyes, fluid can be misdirected via damaged zonules around the iridectomy site. Usually this event has no consequences, but sometimes it can cause aqueous humor misdirection that pushes the $\mathrm{CB}$ process into the sclerotomy site. Surgeons should be fully aware of such a complication, since once the $\mathrm{CB}$ process becomes incarcerated, the narrowed fistula will dysfunction or cause inflammation. Medical treatment and/or surgical/laser intervention might be called for in such cases.
Jeeyoung Kwak, Chong Eun Lee

Department of Ophthalmology, Keimyung University School of Medicine, Daegu, Korea

E-mail (Chong Eun Lee): calzabe@naver.com

\section{Conflicts of Interest}

No potential conflict of interest relevant to this article was reported.

\section{Acknowledgements}

None.

\section{Funding}

The authors received no financial support for this article.

\section{References}

1. Dada T, Bhartiya S, Begum Baig N. Cataract surgery in eyes with previous glaucoma surgery: pearls and pitfalls. $J$ Curr Glaucoma Pract 2013;7:99-105.

2. Husain R, Liang S, Foster PJ, et al. Cataract surgery after trabeculectomy: the effect on trabeculectomy function. Arch Ophthalmol 2012;130:165-70.

3. Saari KM, Heikkila LA. Early post-operative pressure rise with ciliary body incarceration into Watson type trabeculectomy fistula. Acta Ophthalmol Suppl 1987;182:30-3.

4. Melamed S, Frenkel R, Krug JH Jr, Allingham RR. Trabeculectomy with resection of ciliary processes in glaucoma following scleral buckling. Ophthalmic Surg 1988;19:506-7.

5. Wang ZH, Liu GX. A pathological analysis of the causes of failure of filtration operation in 25 glaucomatous eyes. Zhonghua Yan Ke Za Zhi 1994;30:261-3. 